\title{
Hippocampal Atrophy Is Associated with Altered Hippocampus-Posterior Cingulate Cortex Connectivity in Mesial Temporal Lobe Epilepsy with Hippocampal Sclerosis
}

\author{
(D)Y.C. Shih, (D).E. Tseng, DF.-H. Lin, (DH.H. Liou, and DW.Y.I. Tseng
}

O- $\equiv$

\begin{abstract}
BACKGROUND AND PURPOSE: Unilateral mesial temporal lobe epilepsy and hippocampal sclerosis have structural and functional abnormalities in the mesial temporal regions. To gain insight into the pathophysiology of the epileptic network in mesial temporal lobe epilepsy with hippocampal sclerosis, we aimed to clarify the relationships between hippocampal atrophy and the altered connection between the hippocampus and the posterior cingulate cortex in patients with mesial temporal lobe epilepsy with hippocampal sclerosis.
\end{abstract}

MATERIALS AND METHODS: Fifteen patients with left mesial temporal lobe epilepsy with hippocampal sclerosis and 15 healthy controls were included in the study. Multicontrast MR imaging, including high-resolution TTWI, diffusion spectrum imaging, and resting-state fMRI, was performed to measure the hippocampal volume, structural connectivity of the inferior cingulum bundle, and intrinsic functional connectivity between the hippocampus and the posterior cingulate cortex, respectively.

RESULTS: Compared with controls, patients had decreased left hippocampal volume (volume ratio of the hippocampus and controls, $0.366 \% \pm 0.029 \%$; patients, $0.277 \% \pm 0.063 \%$, corrected $P=.002$ ), structural connectivity of the bilateral inferior cingulum bundle (generalized fractional anisotropy, left: controls, $0.234 \pm 0.020$; patients, $0.193 \pm 0.022$, corrected $P=.0001$, right: controls, $0.226 \pm 0.022$; patients, $0.208 \pm 0.017$, corrected $P=.047$ ), and intrinsic functional connectivity between the left hippocampus and the left posterior cingulate cortex (averaged z-value: controls, $0.314 \pm 0.152$; patients, $0.166 \pm 0.062$ ). The left hippocampal volume correlated with structural connectivity positively (standardized $\beta=0.864, P=.001$ ), but it had little correlation with intrinsic functional connectivity (standardized $\beta=-0.329, P=.113)$. On the contralesional side, the hippocampal volume did not show any significant correlation with structural connectivity or intrinsic functional connectivity $\left(F_{2,12}=0.284, P=.757, R^{2}=0.045\right)$.

CONCLUSIONS: In left mesial temporal lobe epilepsy with hippocampal sclerosis, the left inferior cingulum bundle undergoes degeneration in tandem with the left hippocampal volume, whereas intrinsic functional connectivity seems to react by compensating the loss of connectivity. Such insight might be helpful in understanding the development of the epileptic network in left mesial temporal lobe epilepsy with hippocampal sclerosis.

ABBREVIATIONS: $\mathrm{DSI}=$ diffusion spectrum imaging; $\mathrm{GFA}=$ generalized fractional anisotropy; $\mathrm{HS}=$ hippocampal sclerosis; $\mathrm{HV}=$ hippocampal volume; $\mathrm{iCB}=$ inferior cingulum bundle; ;FC = intrinsic functional connectivity; MTLE = mesial temporal lobe epilepsy; $\mathrm{PCC}=$ posterior cingulate cortex; $\mathrm{SC}=$ structural connectivity

$\mathbf{U}^{\prime}$ nilateral mesial temporal lobe epilepsy (MTLE) is a common type of refractory focal epilepsy in which hippocampal sclerosis (HS) is the most frequent pathologic finding. Gray matter

Received June 2, 2016; accepted after revision October 17.

From the Institute of Biomedical Engineering (Y.C.S., F.-H.L.) and Molecular Imaging Center (W.Y.I.T.), National Taiwan University, Taipei, Taiwan; and Institute of Medical Device and Imaging (Y.C.S., C.E.T., W.Y.I.T.), Graduate Institute of Brain and Mind Sciences (H.H.L., W.Y.I.T.), and Departments of Neurology (H.H.L.) and Medical Imaging (W.Y.I.T.), College of Medicine, National Taiwan University, Taipei, Taiwan.

This work was supported by the Ministry of Science and Technology, Taiwan; grant No. MOST104-2325-B-002-040 and MOST 103-2314-B-002-063-MY3; and the Ministry of Economic Affairs, Taiwan; grant No. 101-EC-17-A-19-S1-175.

Results previously presented in part at: Scientific Meeting and Exhibition of the International Society for Magnetic Resonance in Medicine, May 5-11, 2012; Melbourne, Australia; and April 20-26, 2013; Salt Lake City, Utah. atrophy associated with MTLE and HS (MTLE+HS) involves the hippocampus and brain regions outside the mesial temporal lobe. ${ }^{1,2}$ The atrophied GM constitutes a network of regions that are structurally and functionally connected to the epileptogenic mesial temporal region. ${ }^{3}$ Evidence has indicated that an epileptic network consists of both atrophied GM regions ${ }^{2-4}$ and epileptic

Please address correspondence to W.Y.I. Tseng, MD, PhD, Institute of Medical Device and Imaging, National Taiwan University College of Medicine, No. 1, Section 1, Jen-Ai Rd, Taipei 10051, Taiwan; e-mail: wytseng@ntu.edu.tw; H.H. Liou, MD, PhD, Department of Neurology, National Taiwan University Hospital and College of Medicine, No. 7, Chung-Shan South Rd, Taipei 10002, Taiwan; e-mail: hhl@ntu.edu.tw

- Indicates open access to non-subscribers at www.ajnr.org

三 Indicates article with supplemental on-line appendix and table.

Indicates article with supplemental on-line photo.

http://dx.doi.org/10.3174/ajnr.A5039 
seizure-spreading pathways. ${ }^{1}$ Elucidation of altered structural and functional pathways with respect to atrophied GM can provide insight into the pathophysiology of epileptic network development, which might help in identifying biomarkers for the clinical diagnosis and treatment of unilateral MTLE+HS. ${ }^{5}$

The connection between the hippocampus or parahippocampus and the posterior cingulate cortex (PCC) is an afferent pathway of the hippocampus in the Papez circuit, which is altered in MTLE. ${ }^{6}$ Kubota et $\mathrm{al}^{7}$ used corticocortical-evoked potentials to investigate the connectivity between the hippocampus and the PCC in unilateral MTLE. They reported that prominent corticocortical-evoked potential responses were elicited in the hippocampus under PCC stimulation and suggested that electrical stimulation was directly transmitted from the PCC to the hippocampus through a white matter tract. ${ }^{7}$ The inferior cingulum bundle (iCB) is a WM tract projecting from the PCC to the hippocampus or parahippocampus and the entorhinal cortex. ${ }^{8} \mathrm{~A}$ DTI study reported decreased structural connectivity (SC) of the iCB in patients with unilateral MTLE $+\mathrm{HS}^{8}{ }^{8}$ These and the findings of Kubota et al indicated that the $\mathrm{iCB}$ might be a structural pathway transmitting epileptiform discharges into the epileptic network.

Intrinsic functional connectivity (iFC) between brain regions represents the synchronization of blood oxygenation level-dependent signal fluctuations in MR imaging during the resting state. ${ }^{9}$ The resting-state fMRI studies have shown decreased iFC between the epileptogenic hippocampus and the PCC in patients with unilateral MTLE+HS, suggesting that this functional metric represents an altered functional pathway of the epileptic network. ${ }^{10,11}$ Taken together, hippocampus-PCC connectivity (both structural and functional) is a potential pathway of the epileptic network in unilateral MTLE+HS.

Bernasconi et $\mathrm{al}^{12}$ reported that volume loss in the hippocampus was related to epilepsy duration in patients with unilateral MTLE+HS. ${ }^{12}$ Moreover, on the basis of previous human ${ }^{13}$ and animal studies, ${ }^{14}$ they speculated that the progression of hippocampal atrophy leads to the development of an epileptogenic network and aggravates repeated seizures. Therefore, the elucidation of altered structural and functional pathways with respect to hippocampal atrophy can provide insight into the pathophysiology of the epileptic network in MTLE+HS. However, to date, few studies have investigated this relationship.

Therefore, in the present study, we investigated the relationship between hippocampal atrophy and hippocampus-PCC connectivity in unilateral MTLE+HS. We hypothesized that SC and $\mathrm{iFC}$ are altered in patients with unilateral MTLE+HS and that the altered connectivity is associated with hippocampal atrophy, which reflects the pathophysiology of the epileptic network. We used multicontrast MR imaging examinations, including high-resolution T1WI, diffusion spectrum imaging (DSI), and resting-state $\mathrm{fMRI}$ to measure the hippocampal volume (HV), $\mathrm{SC}$ of the $\mathrm{iCB}$, and iFC between the hippocampus and the PCC, respectively.

\section{MATERIALS AND METHODS} Participants

Fifteen patients (mean age, $36.87 \pm 8.79$ years) with left MTLE+HS and 15 healthy controls (mean age, $36.33 \pm 8.73$
Demographic data of patients with left MTLE+HS and healthy controls

\begin{tabular}{lcc}
\hline & $\begin{array}{c}\text { Left } \\
\text { MTLE+HS } \\
(\boldsymbol{n}=15)\end{array}$ & $\begin{array}{c}\text { Controls } \\
(\boldsymbol{n}=15)\end{array}$ \\
\hline Age (mean) (yr) & $36.87 \pm 8.79$ & $36.33 \pm 8.73$ \\
Sex (male/female) & $9: 6$ & $8: 7$ \\
Handedness (left/right) & $0 / 15$ & $0 / 15$ \\
Epilepsy duration (mean) (yr) & $23.47 \pm 10.58$ & - \\
Onset age (mean) (yr) & $13.4 \pm 7.11$ & - \\
Seizure type: (No.) & & - \\
$\quad$ SPS & 1 & \\
CPS & 13 & \\
$\quad$ CPS with secondary generalization & 1 & \\
\hline
\end{tabular}

Note:-CPS indicates complex partial seizure; SPS, simple partial seizure.

years) were recruited for the study. The study was approved by the National Taiwan University Hospital research ethics committee, and all participants provided informed consent before entering the study. The demographics of the participants and detailed criteria for participant recruitment are listed in the Table and the On-line Appendix, respectively.

\section{Data Acquisition}

All participants underwent MR imaging examinations on a 3T MR imaging system (Tim Trio; Siemens, Erlangen, Germany) with a 32-channel phased array head coil. The MR imaging examinations included T1WI, DSI, and resting-state fMRI. T1WI was performed by using a 3D magnetization-prepared rapid acquisition of gradient echo sequence with the following parameters: $\mathrm{TR} / \mathrm{TE}=2000 / 3 \mathrm{~ms}$, flip angle $=9^{\circ}, \mathrm{FOV}=256 \times 192 \times 208$ $\mathrm{mm}^{3}$, and acquisition matrix $=256 \times 192 \times 208$. DSI was performed by using a pulsed gradient spin-echo diffusion EPI sequence with a twice-refocused balanced echo and the following parameters: $\mathrm{TR} / \mathrm{TE}=9600 / 130 \mathrm{~ms}$, flip angle $=90^{\circ}, \mathrm{FOV}=$ $200 \times 200 \mathrm{~mm}^{2}$, acquisition matrix $=80 \times 80 \times 56$, and section thickness $=2.5 \mathrm{~mm}$. A total of 102 diffusion-encoding gradients were applied by using the maximum diffusion sensitivity of 4000 $\mathrm{s} / \mathrm{mm}^{2}$. These encoding gradients corresponded to grid points filled within the half sphere of the $q$-space with a radius of 3 units. ${ }^{15}$ We performed resting-state fMRI by using $2 \mathrm{D}$ gradient-echo EPI with the following parameters: $\mathrm{TR} / \mathrm{TE}=2000 / 24 \mathrm{~ms}$, flip angle $=$ $90^{\circ}, \mathrm{FOV}=256 \times 256 \mathrm{~mm}^{2}$, acquisition matrix $=64 \times 64 \times 34$, section thickness $=3 \mathrm{~mm}$, and 180 volumes per run.

\section{Data Analysis}

The MR imaging procedures and statistical analyses are presented in Fig 1. The volume ratio of the hippocampus (HV/whole-brain volume) was calculated to evaluate the degree of hippocampal atrophy. The whole-brain volume and HV calculations were performed by using FreeSurfer 5.0 software (http://surfer.nmr.mgh. harvard.edu). The details of the analysis procedures have been described elsewhere. ${ }^{16}$ Neuroanatomic labels were automatically assigned to each voxel on the basis of probabilistic information estimated from a manually labeled training set. The HV was obtained in each hemisphere.

We used DSI to evaluate accurate tractography of the iCB because of its ability to resolve crossing fibers, ${ }^{17}$ and we used tractspecific analysis to measure the microstructural integrity along the iCB to index SC. Within each voxel, the 102 samples within the 
Individual T1WI

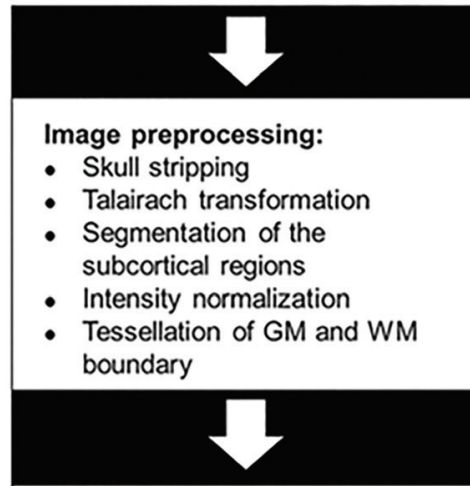

1. Neuroanatomical labeling

\section{HV estimation}
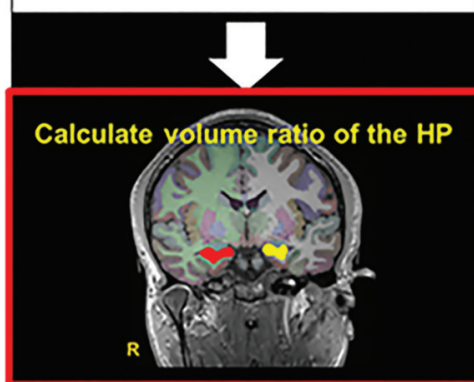

Individual DSI data

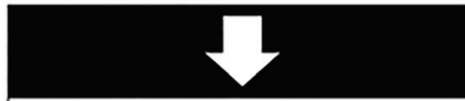

Tractography on DSI template

- ROIs identification from FreeSurfer segmentation labels.

- Reconstruct the $\mathrm{iCB}$

- Shape and specify ROIs

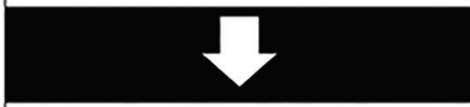

Tractography on individual DSI data

- ROIs transform from DSI template to individual DSI data

- Reconstruct the iCB

- Calculate mean GFA of the iCB

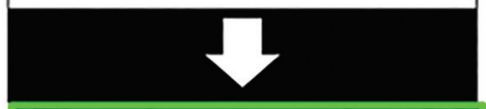

Calculate SC of the iCB

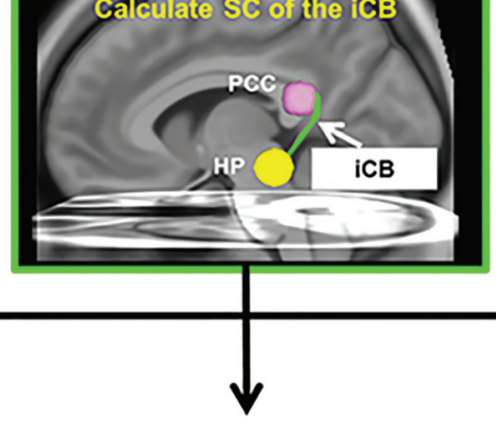

The linear regression model

$$
Y=\beta_{0}+\beta_{1} X_{1}+\beta_{2} X_{2}+\varepsilon
$$

FIG 1. The pipeline of MR imaging and statistical analyses. Multicontrast MR imaging examinations including TIWI, DSI, and resting-state fMRI were performed to measure $\mathrm{HV}$ and $\mathrm{SC}$ of the $\mathrm{iCB}$ and iFC between the hippocampus and the PCC, respectively. A linear regression model was used to evaluate the correlation of HV with SC and iFC indices, where $Y$ is the volume ratio of the hippocampus and $X_{1}$ and $X_{2}$ are the $\mathrm{SC}$ and iFC of the HP-PCC connection, respectively. HP indicates hippocampus; rsfMR, resting-state fMRI.

half sphere of the $q$-space were projected to fill the other half sphere, and 8 corners outside the sphere were filled with zeros. Fourier transform was performed on the $q$-space signal to obtain the diffusion probability density function. ${ }^{17}$ The orientation distribution function was obtained by computing the second moment of the probability density function along each of the 362 radial directions in 6-fold tessellated icosahedrons. To quantify the microstructural integrity, we computed generalized fractional anisotropy (GFA), an index equivalent to fractional anisotropy in DTI ${ }^{18,19}$ for each voxel by using the following formula: (SD of the Orientation Distribution Function)/(Root Mean Square of the Orientation Distribution Function). ${ }^{20}$ The decomposition of the orientation distribution function was performed to determine local tract directions in each voxel, ${ }^{21}$ which were then used for the diffusion tractography of the iCB.
The mean GFA of the left or right iCB was calculated to indicate SC between the hippocampus and the PCC. We performed a template-based tract-specific analysis based on the local tract direction and GFA maps derived from DSI data by using DSI Studio (http://dsi-studio.labsolver.org/). The detailed procedure of tracking the $\mathrm{iCB}$ is described in the On-line Figure.

The Data Processing Assistant for Resting-State fMRI (DPARSF; http://www.rfmri.org/DPARSF) was used to analyze resting-state fMRI data. ${ }^{22}$ The detailed procedures of data preprocessing have been described elsewhere. ${ }^{22}$ The preprocessed data were then filtered by using a bandpass filter $(0.01-0.08 \mathrm{~Hz})$. Finally, nuisance regression was performed to correct the polynomial trend, 6 rigidbody parameters, head-motion scrubbing (power of framewise displacement), and WM and CSF mean signals. We performed seed-based analysis to obtain whole-brain iFC maps by placing a 
spheric seed $(r=6 \mathrm{~mm})$ covering the bilateral PCC. ${ }^{23}$ We computed iFC maps by using voxelwise Pearson correlation coefficients between the time course of each voxel and the averaged time course of the predefined PCC seed. Furthermore, iFC maps were transformed into $Z$-maps by using Fisher $Z$-transform. We used 2-sample $t$ tests to conduct voxelwise group comparison with $Z$-maps between patients with left MTLE+HS and healthy controls. Moreover, we used the spheric ROIs with the radius of 8 $\mathrm{mm}$ centered at peak coordinates to extract $\mathrm{z}$-values. The averaged $z$-values of the spheric ROIs in the hippocampus indicated the iFC between the hippocampus and the PCC.

Statistical analyses were performed by using SPSS, Version 20 (IBM, Armonk, New York). A Mann-Whitney $U$ test was used for comparing age, volume ratio of the hippocampus, and SC between the hippocampus and the PCC (mean GFA of the iCB) between groups. In addition, we performed a Benjamini-Hochberg correction to address the issue of multiple testing. A linear regression model was used to investigate the relationship between the $\mathrm{HV}$ and $\mathrm{SC}$ and the $\mathrm{iFC}$ indices between the hippocampus and the PCC in patients.

\section{RESULTS}

\section{Comparison between Groups}

Healthy controls and patients were well-matched in age $(t(28)=$ $0.167, P=.869$ ), sex, and handedness (Table). Compared with healthy controls, patients had a significant decrease in the volume ratio of the left hippocampus (controls, $0.366 \% \pm 0.029 \%$; patients, $0.277 \% \pm 0.063 \%$; Benjamini-Hochberg-corrected $P=$ .002 ) and no significant difference in the volume ratio of the right hippocampus (controls, $0.387 \% \pm 0.041 \%$; patients, $0.389 \% \pm$ $0.039 \% ; P=.775$ ). In both the left and right iCBs, the mean GFA was significantly lower in patients than in healthy controls (left iCB: controls, $0.234 \pm 0.020$; patients, $0.193 \pm 0.022$; BenjaminiHochberg-corrected $P=.0001$; right iCB: controls, $0.226 \pm$ 0.022; patients, $0.208 \pm 0.017$; Benjamini-Hochberg-corrected $P=.047)$.

The group comparison of iFC maps ( $Z$-maps) revealed 2 peak coordinates in the left hippocampus, indicating decreased iFC between the left hippocampus and the PCC (On-line Table); this was defined by a higher contrast in healthy controls than in patients (peak 1: $T=2.84$, small-volume- corrected $^{24} P=.004$; peak 2: $T=2.39$, small-volume-corrected $P=.012$ ). Moreover, 2 peak coordinates were observed in the right hippocampus, indicating increased iFC between the right hippocampus and the PCC; this was defined by a higher contrast in patients than in healthy controls (peak 3: $T=2.01$, small-volume-corrected $P=$ .027; peak 4: $T=1.91$, small-volume-corrected $P=.028$ ). The averaged $z$-values of the spheric ROIs in the left hippocampus were $0.314 \pm 0.152$ and $0.166 \pm 0.062$ in healthy controls and patients, respectively, whereas those in the right were $0.200 \pm$ 0.130 and $0.291 \pm 0.091$ in healthy controls and patients, respectively.

\section{Relationship between the HV and Hippocampus-PCC SC and iFC Indices in Patients}

In patients' left hemispheres, the overall regression model was significant $\left(F_{2,12}=12.656, P=.001, R^{2}=0.678\right)$. The regression model revealed that $62.6 \%$ (adjusted $R^{2}=0.626$ ) of the volume ratio of the left hippocampus was explained by the mean GFA of the left $\mathrm{iCB}$ and the averaged $\mathrm{z}$-value of the left hippocampus. As presented in Fig $2 A$, the mean GFA of the left iCB positively correlated with the volume ratio of the left hippocampus $(\beta=0.025$, standardized $\beta=0.840, P=.0004$ ). The averaged $\mathrm{z}$-value of the left hippocampus negatively correlated with the volume ratio of the left hippocampus $(\beta=-0.005$, standardized $\beta=-0.509$, $P=.013$, Fig $2 B$ ). In patients' right hemispheres, the regression model was not significant $\left(F_{2,12}=0.284, P=.757, R^{2}=0.045\right)$. Both the mean GFA of the right $\mathrm{iCB}$ and averaged $\mathrm{z}$-value of the right hippocampus did not significantly correlate with the volume ratio of the right hippocampus (Fig $2 C,-D$ ). We found a potential outlier in 1 patient whose averaged z-value of the left hippocampus was larger than 2.5 times the SD. After we removed the outlier, the overall regression model was still significant in the left hemisphere $\left(F_{2,12}=10.212, P=.003, R^{2}=0.650\right.$, adjusted $R^{2}=$ $0.586)$. The correlation was significant between the mean GFA of the left $\mathrm{iCB}$ and the volume ratio of the left hippocampus ( $\beta=$ 0.024 , standardized $\beta=0.864, P=.001$ ); however, the correlation was no longer significant between the averaged $z$-value and the volume ratio of the left hippocampus ( $\beta=-0.004$, standardized $\beta=-0.329, P=.113$ ).

\section{DISCUSSION}

To the best of our knowledge, this is the first study elucidating the relationship between hippocampal atrophy and structural and functional connectivity in patients with left MTLE+HS. We observed that the regression model used was highly significant, even though a potential outlier of data was removed from the model estimation. Specifically, left HV positively correlated with the mean GFA of the left iCB, but it did not correlate with the left iFC. Compared with controls, the HV, SC, and iFC indices were significantly decreased on the lesion side of patients. As discussed below, the association between the HV and its connection to the PCC allowed us to explore the pathophysiology of the epileptic network in unilateral MTLE+HS.

We observed a significant positive correlation between the left $\mathrm{HV}$ and the mean GFA of the left iCB in patients with left MTLE+HS (Fig 2A). Two mechanisms could explain this relationship: First, the iCB may be disrupted because of the excitotoxic effect caused by the spreading of epileptogenic activity through the Papez circuit. ${ }^{25,26}$ The sclerotic hippocampus may generate epileptic activity and spread it through the Papez circuit to multiple epileptogenic regions, such as the mammillary body, thalamus, entorhinal cortex, and cingulate gyrus. The iCB is assumed to receive antegrade epileptic activity from secondary epileptogenic regions and to propagate it back to the hippocampus. Using DTI, Scanlon et $\mathrm{al}^{27}$ demonstrated a positive correlation between the HV and fractional anisotropy in the middle cingulum bundle in patients with unilateral MTLE+HS. Our study further confirmed the correlation in the $\mathrm{iCB}$ on the lesion side. Another possible mechanism is that hippocampal cellular death caused by HS may affect the extrahippocampal WM, resulting in the axonal degeneration of the $\mathrm{iCB} .^{28}$ This interpretation is based on an assumption that the $\mathrm{iCB}$ directly connects to the hippocampus. Kubota et $\mathrm{al}^{7}$ used corticocortical-evoked poten- 

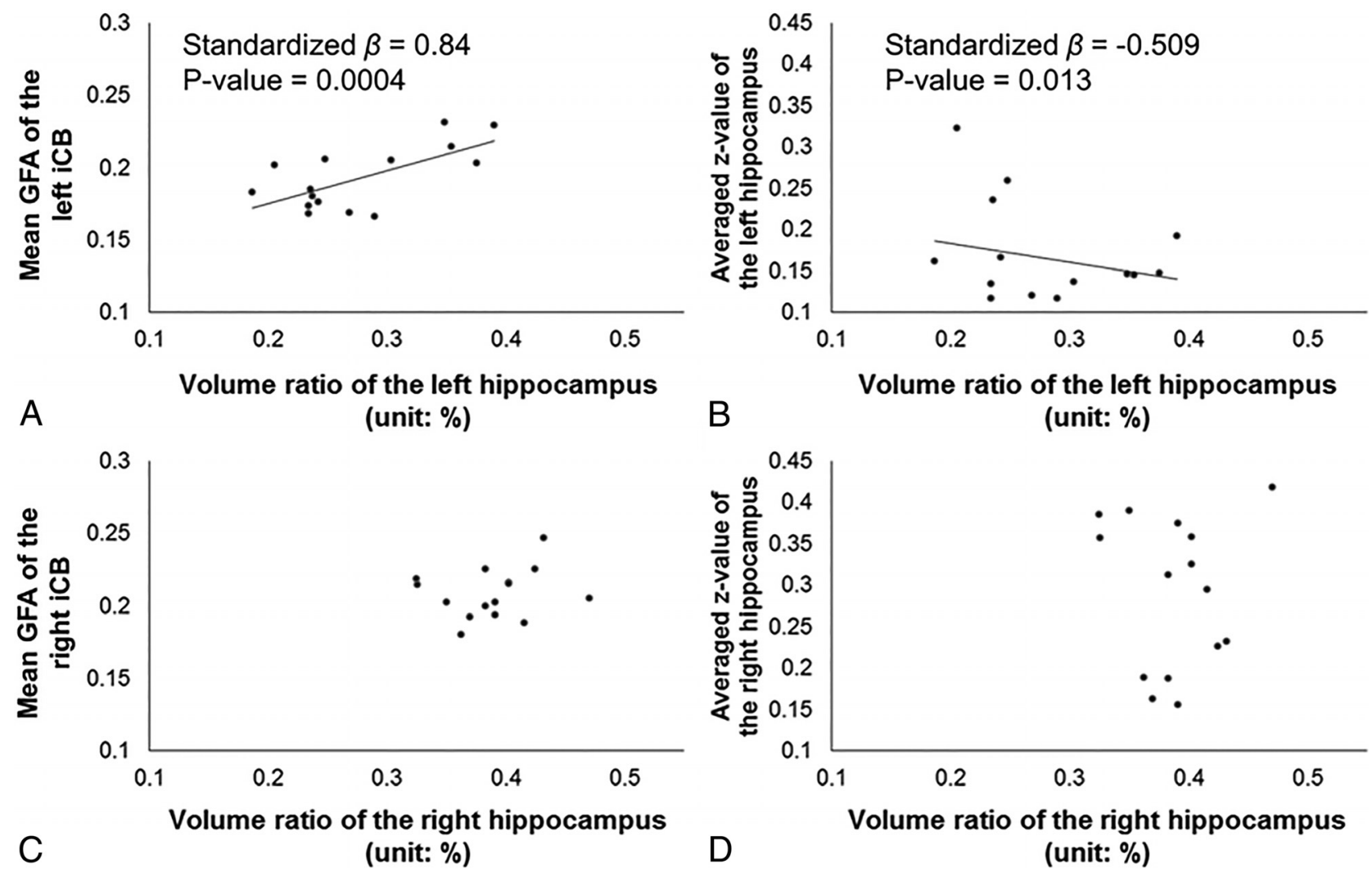

FIG 2. Relationships between HV and hippocampus-PCC SC and iFC indices in patients. We found a significant relationship between the volume ratio of the hippocampus ( $x$-axis) and the mean GFA of the $\mathrm{iCB}$ (y-axis) (A) and between the volume ratio of the hippocampus ( $x$-axis) and averaged $z$-value of the hippocampus ( $y$-axis) $(B)$ in the left hemisphere in patients with left MTLE+HS, whereas there were no correlations in the right hemisphere $(C$ and $D)$.

tials for electrical stimulation studies and reported that hippocampal stimulation induced a prominent response in the PCC and, conversely, that the hippocampus was elicited by PCC stimulation in patients with unilateral MTLE. Moreover, on the basis of corticocortical-evoked potentials, they speculated strong functional connectivity between the hippocampus and the PCC through the iCB. Therefore, hippocampal cellular death caused by HS following epileptogenesis might directly degrade the SC of the iCB.

Recent DTI studies have reported inconsistent results on the relationship between GM atrophy and impaired hippocampal WM structure in patients with unilateral MTLE + HS. ${ }^{3,29}$ Ellmore et $\mathrm{al}^{29}$ reported that the number of hippocampal fibers derived from probabilistic tractography positively correlated with the HV. Bonilha et $\mathrm{al}^{3}$ investigated the relationship between the mean fractional anisotropy or mean diffusivity of perihippocampal fibers and the GM density of several brain regions in unilateral MTLE+HS; they did not find correlations between the HV and diffusion properties (fractional anisotropy and mean diffusivity) of perihippocampal fibers. With the advantage of DSI in resolving crossing fibers, we used tract-specific analysis to calculate the SC of the iCB and observed strong coupling with hippocampal atrophy in left MTLE+HS.

We observed decreased mean GFA of the left iCB in patients with left MTLE+HS. Our finding is similar to that of a previous DTI study on unilateral MTLE+HS. ${ }^{8}$ In addition to the left $\mathrm{iCB}$, decreased fractional anisotropy was observed in the right $\mathrm{ICB}$ in patients with left MTLE+HS, ${ }^{8}$ which is consistent with our results. Their findings and ours support the idea that the SC of the bilateral iCB is altered in unilateral MTLE+HS; this alteration suggests that unilateral MTLE+HS is an extensive network disease. ${ }^{30}$ Moreover, we did not observe a significant relationship between the right $\mathrm{HV}$ and mean GFA of the right iCB. According to our results, the right $\mathrm{HV}$ of patients was comparable with that of controls. Therefore, the mean GFA of the right iCB might be decreased in patients because of the excitotoxic injury caused by seizure spread. ${ }^{25,26}$

We found decreased iFC between the left hippocampus and the left PCC in patients with left MTLE+HS (On-line Table). Our results are consistent with those of previous studies that reported decreased iFC between the PCC and the epileptogenic hippocampus or mesial temporal lobe in patients with left MTLE+HS. ${ }^{10,31}$ In addition, we observed increased $\mathrm{iFC}$ on the right side in the same group of patients (On-line Table). Bettus et $\mathrm{al}^{32}$ investigated iFC between the hippocampus and other limbic structures on the healthy side in a group of patients with unilateral $\mathrm{MTLE}^{32}$; they reported increased iFC between the right hippocampus and the right temporal pole and right amygdala in patients with left MTLE. Their and our results suggest that increased iFC of the contralesional hippocampus is a compensatory response to decreased hippocampal connectivity on the lesion side.

Unlike strong coupling between the left HV and the mean GFA of the left iCB found in patients, the left HV showed little correlation with left iFC, especially after removal of an outlier (Fig 2B). 
A study hypothesized that increased hippocampal activation or connectivity in patients with hippocampal injury might be a compensatory response relying on brain plasticity or an abnormal brain function reflecting pathologic changes. ${ }^{33}$ In the same vein, our negative findings may imply dynamic changes of iFC in response to hippocampal atrophy or iCB degeneration. The left iFC in patients being generally decreased implies a decompensated, rather than compensated, status of iFC.

This study has some limitations. First, although we investigated the relationship between hippocampal atrophy and impaired hippocampus-PCC connectivity, the cross-sectional study design precludes us from determining a causal relationship. Second, the sample size in this study was relatively small. We included a small cohort of patients with unilateral MTLE who had only a left atrophic hippocampus. to reduce the influence of the heterogeneity within the populations of patients with MTLE+HS. ${ }^{34,35}$ The strict inclusion criteria of subject selection limited our sample size. Nonetheless, our findings still remained significant after adjusting for multiple tests. Third, considering differences in the epileptogenic and neurophysiologic processes between left and right MTLE + HS, ${ }^{36}$ our results might not be applicable to right, bilateral, or other subtypes of MTLE+HS.

\section{CONCLUSIONS}

We performed multicontrast MR imaging examinations and demonstrated that patients with left MTLE+HS had decreased $\mathrm{HV}, \mathrm{SC}$, and iFC indices on the lesion side. The left HV was positively correlated with SC, but not iFC. Such findings reflect the development of a degenerative fiber pathway with functional reactivity in an epileptic network. Such insight might be helpful in understanding the pathophysiology of the epileptic network.

\section{ACKNOWLEDGMENTS}

We thank all participants who joined this study and research assistants who helped collect and archive the data.

Disclosures: Fa-Hsuan Lin-RELATED: Grant: Ministry of Science and Technology, Taiwan, Comments: MOST 104-2314-B-002-238, MOST 103-2628-B-002-002-MY3.* *Money paid to the institution.

\section{REFERENCES}

1. Chan S, Erickson JK, Yoon SS. Limbic system abnormalities associated with mesial temporal sclerosis: a model of chronic cerebral changes due to seizures. Radiographics 1997;17:1095-110 CrossRef Medline

2. Riederer F, Lanzenberger R, Kaya M, et al. Network atrophy in temporal lobe epilepsy: a voxel-based morphometry study. Neurology 2008;71:419-25 CrossRef Medline

3. Bonilha L, Edwards JC, Kinsman SL, et al. Extrahippocampal gray matter loss and hippocampal deafferentation in patients with temporal lobe epilepsy. Epilepsia 2010;51:519-28 CrossRef Medline

4. Spencer SS. Neural networks in human epilepsy: evidence of and implications for treatment. Epilepsia 2002;43:219-27 CrossRef Medline

5. Gleichgerrcht E, Kocher M, Bonilha L. Connectomics and graph theory analyses: novel insights into network abnormalities in epilepsy. Epilepsia 2015;56:1660-68 CrossRef Medline

6. McDonald CR, Ahmadi ME, Hagler DJ, et al. Diffusion tensor imaging correlates of memory and language impairments in temporal lobe epilepsy. Neurology 2008;71:1869-76 CrossRef Medline
7. Kubota Y, Enatsu R, Gonzalez-Martinez J, et al. In vivo human hippocampal cingulate connectivity: a corticocortical evoked potentials (CCEPs) study. Clin Neurophysiol 2013;124:1547-56 CrossRef Medline

8. Liacu D, Idy-Peretti I, Ducreux D, et al. Diffusion tensor imaging tractography parameters of limbic system bundles in temporal lobe epilepsy patients. J Magn Reson Imaging 2012;36:561-68 CrossRef Medline

9. Fox MD, Raichle ME. Spontaneous fluctuations in brain activity observed with functional magnetic resonance imaging. Nat Rev Neurosci 2007;8:700-11 CrossRef Medline

10. McCormick C, Quraan M, Cohn M, et al. Default mode network connectivity indicates episodic memory capacity in mesial temporal lobe epilepsy. Epilepsia 2013;54:809-18 CrossRef Medline

11. James GA, Tripathi SP, Ojemann JG, et al. Diminished default mode network recruitment of the hippocampus and parahippocampus in temporal lobe epilepsy. J Neurosurg 2013;119:288-300 CrossRef Medline

12. Bernasconi N, Natsume J, Bernasconi A. Progression in temporal lobe epilepsy: differential atrophy in mesial temporal structures. Neurology 2005;65:223-28 CrossRef Medline

13. Bragin A, Wilson CL, Engel J Jr. Chronic epileptogenesis requires development of a network of pathologically interconnected neuron clusters: a hypothesis. Epilepsia 2000;41(suppl 6):S144-52 CrossRef Medline

14. Roch C, Leroy C, Nehlig A, et al. Magnetic resonance imaging in the study of the lithium-pilocarpine model of temporal lobe epilepsy in adult rats. Epilepsia 2002;43:325-35 CrossRef Medline

15. Kuo LW, Chiang WY, Yeh FC, et al. Diffusion spectrum MRI using body-centered-cubic and half-sphere sampling schemes. J Neurosci Methods 2013;212:143-55 CrossRef Medline

16. Fischl B, van der Kouwe A, Destrieux C, et al. Automatically parcellating the human cerebral cortex. Cereb Cortex 2004;14:11-22 CrossRef Medline

17. Wedeen VJ, Hagmann P, Tseng WY, et al. Mapping complex tissue architecture with diffusion spectrum magnetic resonance imaging. Magn Reson Med 2005;54:1377-86 CrossRef Medline

18. Fritzsche KH, Laun FB, Meinzer HP, et al. Opportunities and pitfalls in the quantification of fiber integrity: what can we gain from Qball imaging? Neuroimage 2010;51:242-51 CrossRef Medline

19. Takagi T, Nakamura M, Yamada M, et al. Visualization of peripheral nerve degeneration and regeneration: monitoring with diffusion tensor tractography. Neuroimage 2009;44:884-92 CrossRef Medline

20. Tuch DS. Q-ball imaging. Magn Reson Med 2004;52:1358-72 CrossRef Medline

21. Yeh FC, Tseng WY. Sparse solution of fiber orientation distribution function by diffusion decomposition. PLoS One 2013;8:e75747 CrossRef Medline

22. Chao-Gan Y, Yu-Feng Z. DPARSF: A MATLAB toolbox for "pipeline" data analysis of resting-state fMRI. Front Syst Neurosci 2010; 4:13 CrossRef Medline

23. Fox MD, Snyder AZ, Vincent JL, et al. The human brain is intrinsically organized into dynamic, anticorrelated functional networks. Proc Natl Acad Sci U S A 2005;102:9673-78 CrossRef Medline

24. Worsley KJ, Marrett S, Neelin P, et al. A unified statistical approach for determining significant signals in images of cerebral activation. Hum Brain Mapp 1996;4:58-73 Medline

25. de Lanerolle NC, Lee TS. New facets of the neuropathology and molecular profile of human temporal lobe epilepsy. Epilepsy Behav 2005;7:190-203 CrossRef Medline

26. Williamson A, Spencer SS, Spencer DD. Depth electrode studies and intracellular dentate granule cell recordings in temporal lobe epilepsy. Ann Neurol 1995;38:778-87 CrossRef Medline

27. Scanlon C, Mueller SG, Cheong I, et al. Grey and white matter abnormalities in temporal lobe epilepsy with and without mesial temporal sclerosis. J Neurol 2013;260:2320-29 CrossRef Medline

28. De Vos KJ, Grierson AJ, Ackerley S, et al. Role of axonal transport in 
neurodegenerative diseases. Ann Rev Neurosci 2008;31:151-73 CrossRef Medline

29. Ellmore TM, Pieters TA, Tandon N. Dissociation between diffusion MR tractography density and strength in epilepsy patients with hippocampal sclerosis. Epilepsy Res 2011;93:197-203 CrossRef Medline

30. Concha L, Beaulieu C, Gross DW. Bilateral limbic diffusion abnormalities in unilateral temporal lobe epilepsy. Ann Neurol 2005;57: 188-96 CrossRef Medline

31. Doucet G, Osipowicz K, Sharan A, et al. Extratemporal functional connectivity impairments at rest are related to memory performance in mesial temporal epilepsy. Hum Brain Mapp 2013;34: 2202-16 CrossRef Medline

32. Bettus G, Guedj E, Joyeux F, et al. Decreased basal fMRI functional connectivity in epileptogenic networks and contralateral compen- satory mechanisms. Hum Brain Mapp 2009;30:1580-91 CrossRef Medline

33. La Joie R. Toward a better understanding of the injured hippocampus: multimodal imaging in functionally relevant substructures. J Neurosci 2014;34:10793-94 CrossRef Medline

34. Bonilha L, Martz GU, Glazier SS, et al. Subtypes of medial temporal lobe epilepsy: influence on temporal lobectomy outcomes? Epilepsia 2012;53:1-6 CrossRef Medline

35. Thom M, Mathern GW, Cross JH, et al. Mesial temporal lobe epilepsy: how do we improve surgical outcome? Ann Neurol 2010; 68:424-34 CrossRef Medline

36. Zhang Z, Lu G, Zhong Y, et al. Altered spontaneous neuronal activity of the default-mode network in mesial temporal lobe epilepsy. Brain Res 2010;1323:152-60 CrossRef Medline 